\title{
Design and Construction of Precision Tooling for the Construction of Resistive Strip Micromegas Detectors for the ATLAS Small Wheel Upgrade Project
}

\author{
Ralph Müller, on behalf of the ATLAS Muon Collaboration
}

\begin{abstract}
Mechanical precision is a key aspect of the $2 \mathrm{~m}^{2}$ high-rate capable Micromegas detectors for the upgrade of the Small Wheels of the ATLAS muon spectrometer. The Micromegas will be built as quadruplets with cathodes and strip-anodes made of stable honeycomb sandwiches.

To achieve the required single plane resolution below $100 \mu \mathrm{m}$ the deviation from planarity of a single detector plane must not exceed $80 \mu \mathrm{m}$ in direction perpendicular to the precision coordinate. The global position of the readout strips has to be within $30 \mu \mathrm{m}$ for a single readout-plane of three PCBs, as well as between all four planes of a quadruplet.

Precision tooling is used for the correct positioning of readout PCBs and readout sandwich planes. For quality control of the planarity of the sandwich planes a laser distance sensor combined with a coordinate measurement system has been developed. Deviation from planarity below $10 \mu \mathrm{m}$ can be easily resolved. We will present key features of the challenging construction procedure to achieve this high level of precision as well as our alignment strategies. This includes the construction and commissioning of a $2.5 \mathrm{~m}^{2}$ lightweight rigid structure (stiffback), which has an overall planarity below $15 \mu \mathrm{m}$ RMS and the measurement of the the blow up of outer planes of a quadruplet due to 2 mbar overpressure of the $\mathrm{Ar} \mathrm{CO}_{2}$ detector gas, the standard situation in ATLAS.
\end{abstract}

\section{INTRODUCTION}

At the LHC storage ring at CERN today protons are colliding with a center of mass energy $\sqrt{s}=13 \mathrm{TeV}$. To investigate and search for rare processes in the field of particle physics the accelerator luminosity steadily increases after the second long shutdown of the LHC in 2019 to $2-3 \cdot 10^{34} \mathrm{~cm}^{-2} \mathrm{~s}^{-1}$. At the inner endcap region of the ATLAS muon spectrometer [1], the Small Wheel, the current detector technologies will exceed their rate limit and need to be replaced during long shut down 2 by Micromegas detectors (Micro Mesh Gaseous Structures) [2] and sTGC detectors (small Strip Thin Gap Chamber) [3]. These detectors will be included in the level one trigger as well[4]. This will allow for determination at trigger level whether the muon track originates from the interaction point and to sort out events faking muons and thus occupying bandwidth of the data stream[5]. Alternatively to increase the transverse momentum threshold would cut off large regions relevant for physics analyses.

In the following the challenging construction procedure to

Manuscript received November 19, 2015.

R. Müller, Ludwig-Maximilians-Universität, Munich, Germany (telephone: +49 89289 14160, e-mail: ralph.mueller@physik.uni-muenchen.de). build Micromegas detectors will be explained and the quality of accomplished components will be shown.

\section{Working PRINCIPLE OF RESISTIVE StRIP Micromegas Detectors}

Micromegas are planar gaseous detectors consisting of a micro pattern anode, a micro mesh and a cathode plane. A traversing muon ionizes the gas mixture ( $\left.\mathrm{Ar}: \mathrm{CO}_{2} 93: 7 \mathrm{vol} \%\right)$ in the detector. The electrons drift towards the stainless steel micro mesh. The region between cathode and mesh is called drift region with an electrical field of $E_{\text {drift }}=0.6-1 \frac{\mathrm{kV}}{\mathrm{cm}}$. The volume below the drift region is called amplification region. Due to the fifty times higher field of $E_{a m p} \approx 43 \frac{\mathrm{kV}}{\mathrm{cm}}$ in this volume, the drifted electrons are pulled through the mesh and start an electron avalanche towards the anode. The anode is a layer of parallel strips with a resistivity of $\approx 10 \frac{M \Omega}{\mathrm{cm}}$. This design makes the Micromegas high rate capable[6]. Charge accumulated on the resistive strips couples capacitively to the copper readout strips underneath. The height of $128 \mu \mathrm{m}$ between mesh and anode is ensured by precise insulating micro pillars. The pitch of the copper strips is typically below $0.5 \mathrm{~mm}$. A schematic drawing is shown in figure 1



Fig. 1. Cross-section of a resistive strip Micromegas detector.

\section{New Small Wheel Sector Design}

The New Small Wheel (NSW) will be subdivided into eight small and eight large sectors, see figure 2 


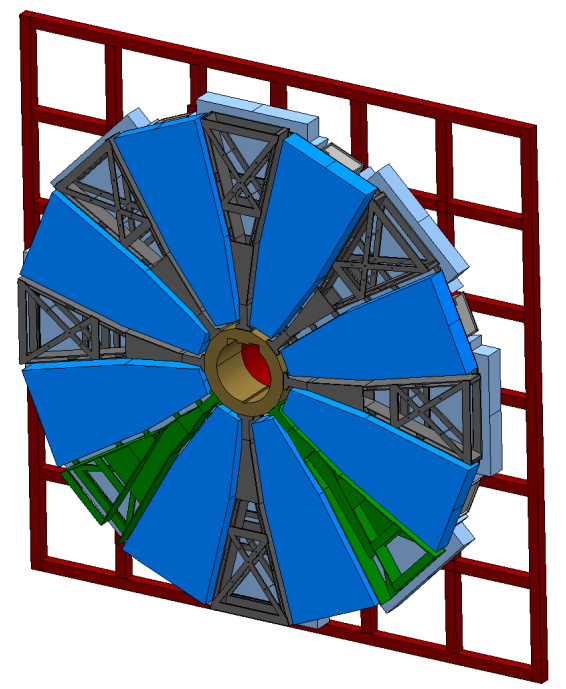

Fig. 2. Sector separation of the New Small Wheel. Eight small and eight large overlapping and alternating sectors form a disk like structure.

In radial direction each sector is furthermore subdivided into two trapezoidal geometries, as shown in figure 3

Since the modules are $2-3 \mathrm{~m}^{2}$ in size, the anode and cathode plane can't be made out of a single PCB. The numbers from 1 to 8 in figure 3 shows the subdivision in individual PCBs.

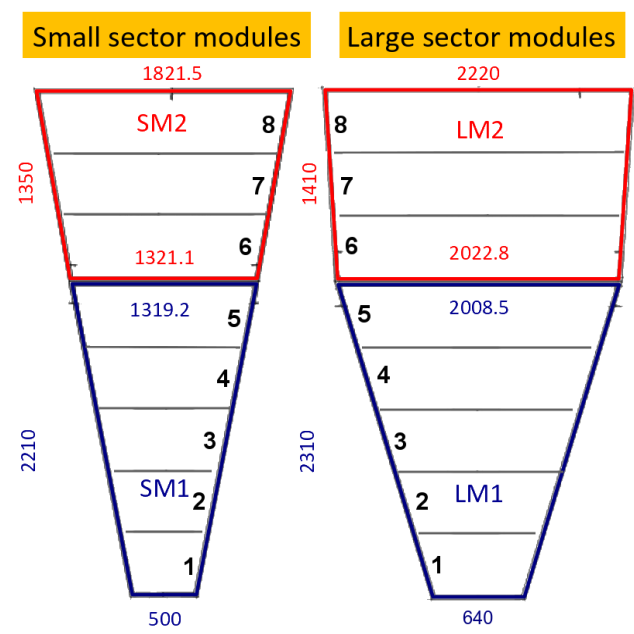

Fig. 3. The Micromegas part of the New Small Wheel is split in radial direction into two trapezoidal geometries for each sector. Due to the fact that industrial produced PCBs are limited in size five or three PCBs form the inner or outer modules.

The SM2 module, which is discussed in detail in this work has a size of $2 \mathrm{~m}^{2}$.

For redundancy four Micromegas active planes are realized by five planar sandwich panels being assembled to form quadruplets. To reach the achieved single plane spatial resolution of $100 \mu \mathrm{m}$ each panel needs to have a planarity better than $80 \mu \mathrm{m}$. The anode strips must be straight and parallel within $30 \mu \mathrm{m}$. This holds for strips on the same PCB as well as for different PCBs and different detector planes.

\section{Panel Construction Using a Stiffback}

The following section describes the construction method used for SM2 sandwich panel production.

\section{A. Two Step Gluing Technique}

A structurally stiff sandwich panel consists of an internal stiffening structure of extruded aluminum bars and aluminum honeycomb sandwiched by $0.5 \mathrm{~mm}$ thick FR4 PCB material The construction takes place in two steps, see figure 4
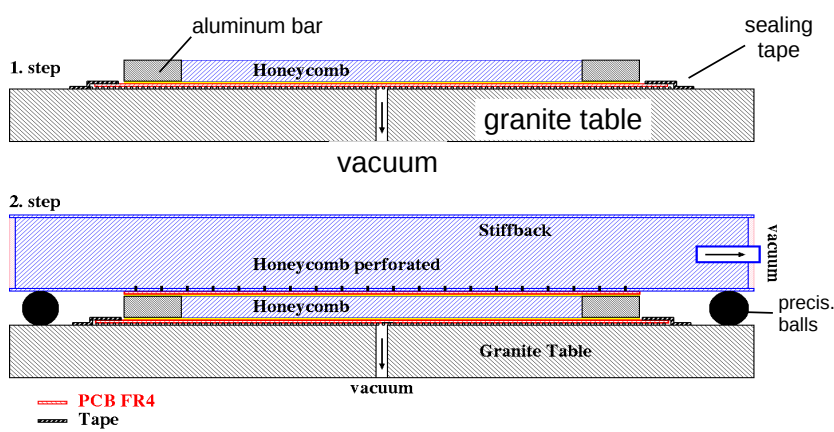

Fig. 4. Scheme of the two step gluing procedure to produce a panel.

In both steps the three SM2 boards are placed strip-side down on a flat granite table. The planarity of the table is below $6 \mu \mathrm{m}$. The boards are aligned precisely as described in section IV-B After alignment the boards are sucked by vacuum to the granite table, such that the board adapts to the flatness of the table. A stiffening structure of extruded bars and honeycomb is precisely aligned and then glued onto the PCBs. The result can be seen in figure 5

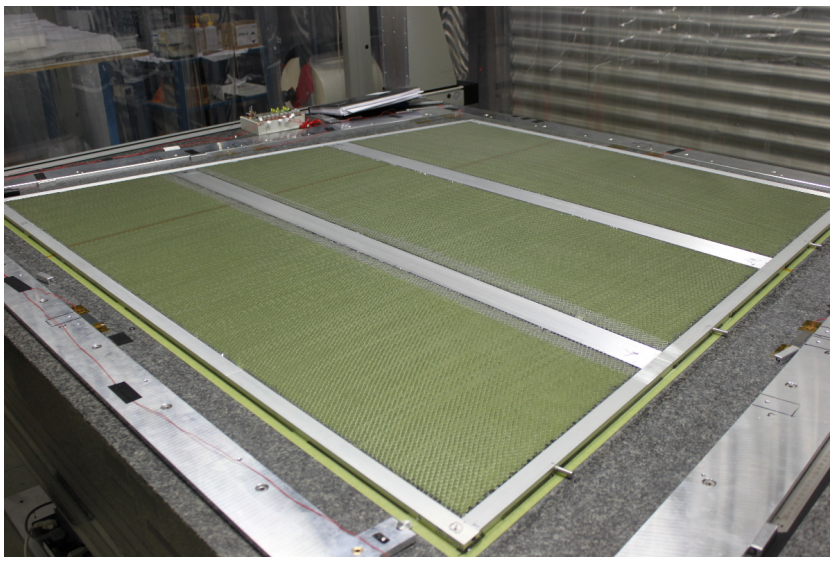

Fig. 5. First step of the gluing process after alignment of PCBs and aluminum bars. In the next step a vacuum bag will be put over the assembly to press everything homogeneously onto the table.

To press the bars and the honeycomb onto the PCB boards a foil covers the assembly to evacuate it from underneath. The glue needs to cure for $10 h$ before the vacuum can be turned off and the second step can be started.

For this the first half-panel is removed from the granite table and a second set of PCBs is aligned on the table identically to the first step. The first half-panel is meanwhile sucked to 
the lightweight flat rigid structure, called stiffback. Glue is dispensed on the second set of PCB and the half-panel on the stiffback is lowered onto the PCBs.

To ensure the parallelism of the first and second side, the stiffback is lying on eight identical precision distance pieces (aluminum cubes) which are defining the height of the panel. A picture taken during the curing of the second side can be seen in figure 6.

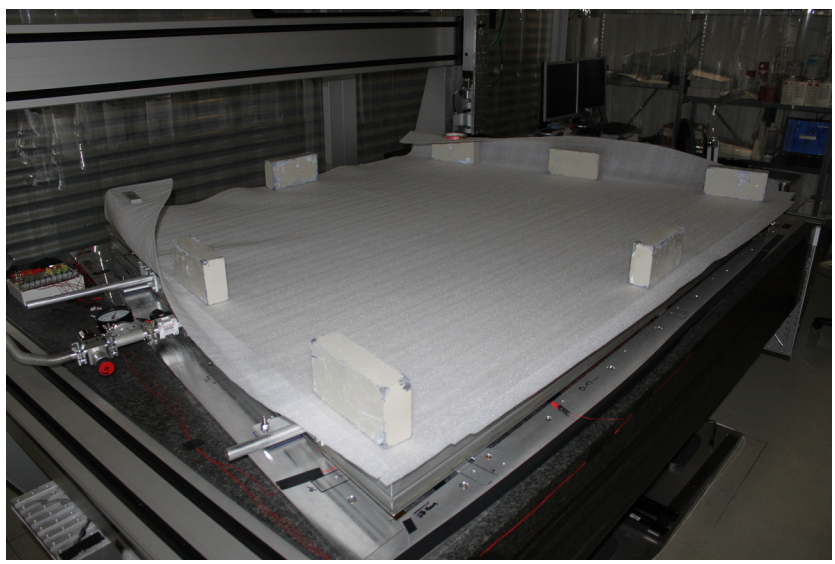

Fig. 6. Second step of the gluing process. Bricks of lead provide additional pressure on top of the eight distance pieces to guarantee proper contact.

Eight bricks of lead, as shown in figure 6 provide additional pressure on top of the distance pieces. After another $10 h$ of curing the gluing process is finished.

\section{B. Precise Alignment of the Readout Strips for the Gluing Process}

Before the gluing process can be started, the PCBs need to be prepared by use of precision alignment.

During the etching of the copper strips on the readout boards also target markers are etched at precise positions relative to the anode strips, see figure 7 .

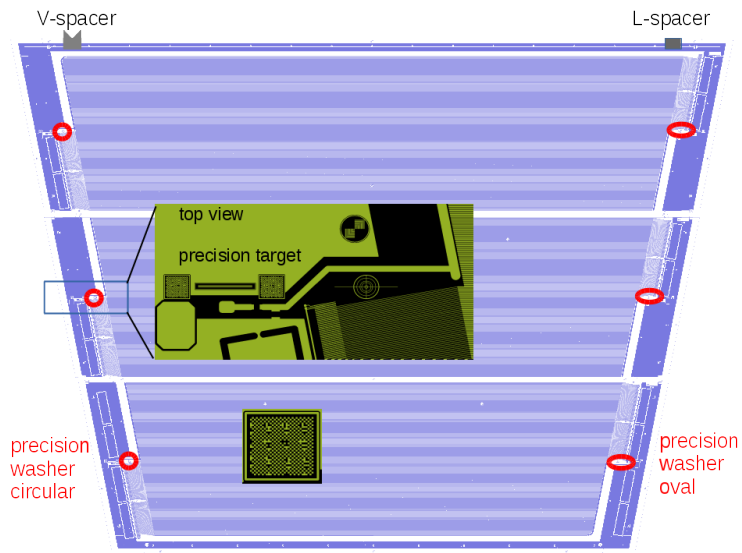

Fig. 7. Three PCBs (violet) with the position of markers highlighted with red circles. A zoom (green) shows several markers which can be used.

Aligning the markers on different boards to each other results in parallel readout strips.

Illuminating the PCB from the back the marker can be seen and thus it can be targeted through the PCB with a telecentric and the exact center of it is determined. Such a camera has special optics to minimize parallax errors.

Using a fine tunable 2D manipulator a brass ring with a precise hole (H7 - accuracy), a so called washer, is aligned to the center of the marker with an accuracy of $<10 \mu \mathrm{m}$. The washer is fixated with fast UV-curing glue. A picture of the setup is shown in figure 8 and the image of the telecentric camera with a well aligned washer is shown in figure 9

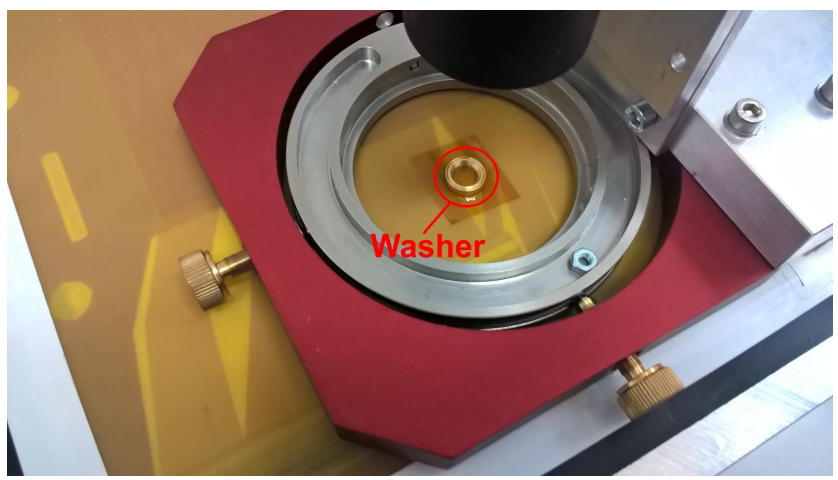

Fig. 8. Washer placed on a PCB with respect to the precision marker.

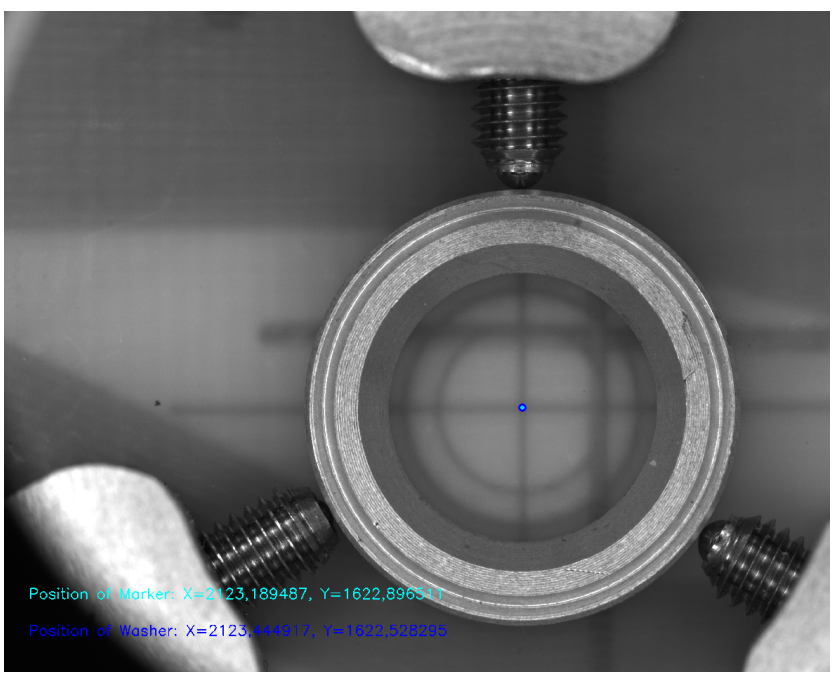

Fig. 9. Image seen by a telecentric camera. The position of the marker is defined by the crossing of the two lines. The position of the washer is defined by the center of the innermost circular edge of the washer, indicated with the blue spot. The washer can now be finely positioned with a 2D manipulator, until the cross center and the blue spot coincide as shown in this image.

The prepared boards are then placed on the granite table and aligned roughly ( $1 \mathrm{~mm}$ accuracy) to an aluminum frame mounted on the table, yellow frame in figure 10.

Another frame of aluminum (red in figure 10 is now lowered to the three PCBs. It has six precision pins (g6 - accuracy) at positions where the washers on the PCBs are placed. The pins fit exactly in the washers and thus aligning all three boards to each other.

The alignment frame itself has on its long side precise Vshaped and line-shaped inserts at positions where the outer 
aluminum frame has fingers with precision cylinders. This is used to align the frame with the boards to a global reference system.

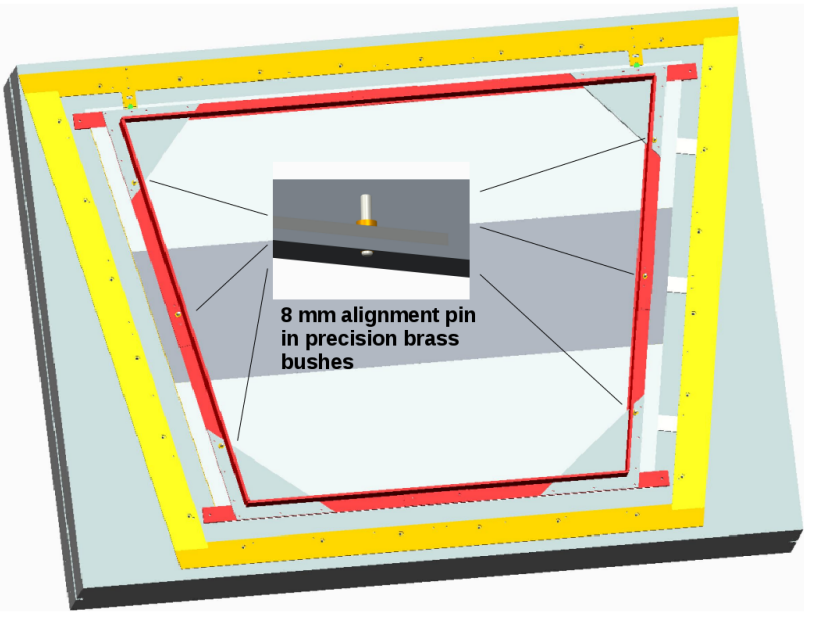

Fig. 10. Aluminum frame mounted permanently on the granite table (yellow) and alignment frame with precision pins (red) for PCB alignment.

Vacuum is applied underneath the boards in order to fix them on the table. The alignment frame is now lifted off the boards. The sides, where the PCBs fit together are sealed with thin Kapton tape while the outer sides are sealed with standard tape. The first side is now perfectly aligned and the gluing process can start.

In addition to the process described in section IV-A a V-shaped and line-shaped insert are glued to the largest board touching the reference fingers of the outer aluminum frame. This will be needed for the second gluing step to align the first half panel to the second set of PCBs.

\section{LASER TRIANGULATION}

To test the flatness of the panels a laser triangulation sensor is mounted on a coordinate measurement machine, see figure 11

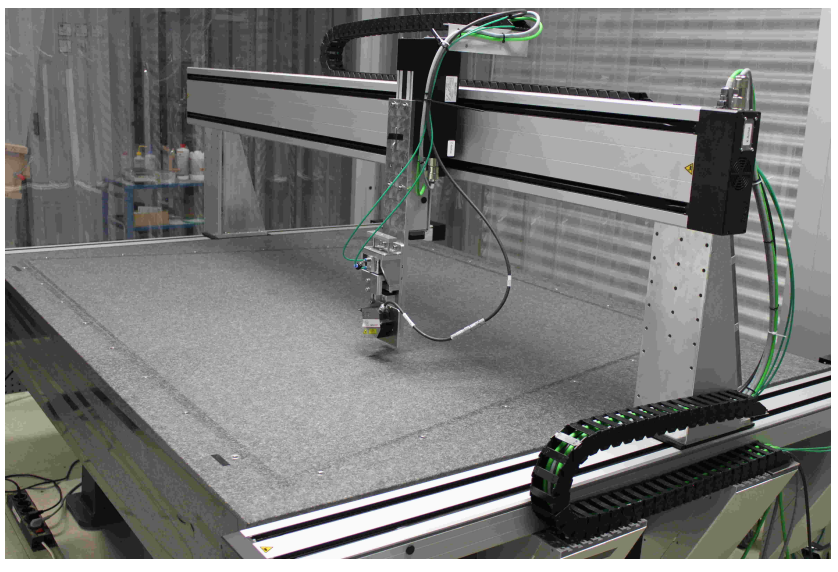

Fig. 11. Coordinate measurement machine (CMM) mounted on a granite table.

This method guarantees a fast scan of the surface without touching it, hence the sensitive readout surfaces, especially the mesh supporting pillars, are secure. The principle of laser triangulation is explained in figure 12

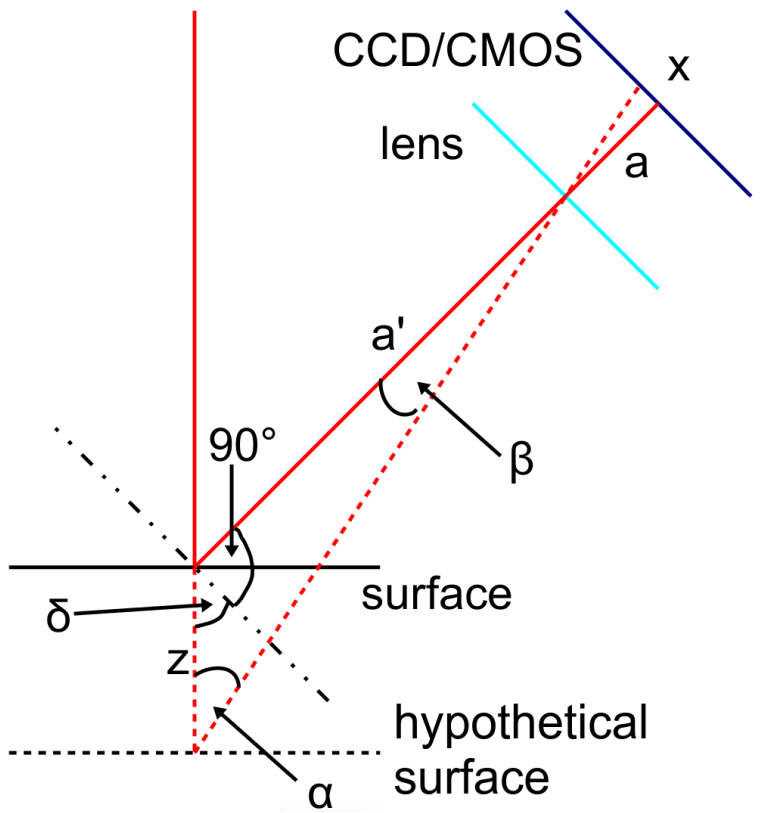

Fig. 12. Scheme of the triangulation principle.

A standard laser triangulator projects a beam on to a surface. The beam is diffusely reflected by the surface and a part of the light goes into a lens in the triangulator where it is focused onto a position sensitive CCD or CMOS sensor, red solid line in figure 12. If the surface is a bit lower than in the case before, the light is focused on a different point on the chip in the sensor, red dashed line in figure 12. The difference between a point on the CCD and a reference point $x$ is used to calculate the variation in height compared to the reference $z$. Using trigonometric identities a formula to calculate $z$ can be obtained.

$$
z=\frac{a^{\prime}}{\frac{a}{x} \cos \delta-\sin \delta}
$$

This formula only depends on $x$ since the parameters $a, a^{\prime}$, and $\delta$ determine the reference surface.

The entire measurement system has a resolution better than $10 \mu \mathrm{m}$.

The sensor used has an additional mode, where the beam hits the surface under a fixed inclination. In addition to the diffuse reflected light, a significant amount of light gets specularly reflected. This mode gives better results for transparent and semi transparent surfaces, because the specular reflection happens on the material whereas diffuse reflection takes place also inside the material. Since the surfaces to be measured are almost all of the semi transparent type, this feature is important. To further improve the result, a blue sensor [7] $(\lambda=405 \mathrm{~nm})$ is used because light with a shorter wavelength gets scattered earlier in semitransparent material.

Since the CMM has no compensation of internal deformations, the granite table is used as reference surface. The table has a certified planarity better $6 \mu \mathrm{m}$. The deformation of the $\mathrm{CMM}$ is constant for every measurement, thus calculating the 
difference between a measured surface and the granite surface eliminates the influence of the CMM and the result is the true topology of the surface to be tested.

\section{Flatness of Glued Surfaces}

The following chapter shows results of the recently produced surfaces. These are test panels to study the gluing procedure and the quality of the surfaces.

\section{A. Topology of the Stiffback}

At first the topology of the stiffback is presented. We produced the stiffback in a similar two step procedure as the panels but without using a stiffback for the second gluing step. The second side is the flat side and will be used for panel production.

Figure 13 shows the flatness of the second side of the stiffback.

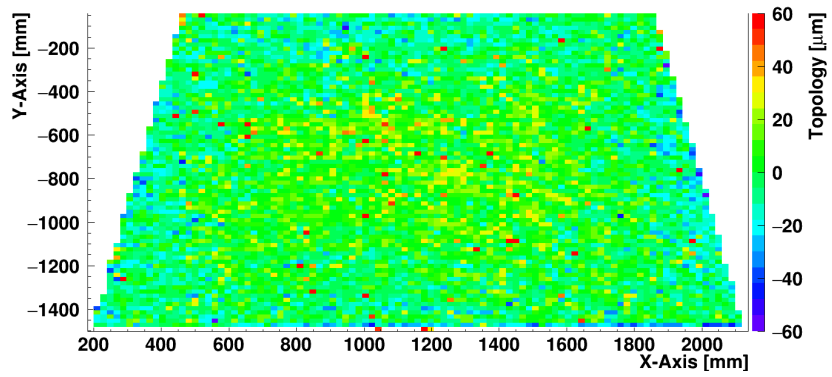

Fig. 13. Topology scan of the second side of the stiffback, which will be used for panel production.

It has an RMS of $14.3 \mu \mathrm{m}$ and $\Delta_{\text {Min-Max }}=80 \mu \mathrm{m}$. This fulfills the requirements imposed to the stiffback by the collaboration. Therefore it will be used for module zero and series production.

The red and violet spots in the histogram originate from the granite reference measurement. Light is not perfectly reflected at the granite surface when it hits transparent crystals.

\section{B. Topology of Test Gluing}

The following plots show the two sides of a panel with bare FR4 on each side. During measurement the panel was sucked to the granite table with vacuum. The topologies are plotted in figures 14 and 15 while the residuals of both surfaces are superimposed in figure 16

Both sides show a deepening in the center. This is an effect of the vacuum suction. The vacuum suction causes the side facing the granite table to be pressed flat, hence the total non planarity of the panel is transferred to the side facing the laser sensor. It can only happen when the non planarity is minor. The RMS of both sides is below $25 \mu \mathrm{m}$ and for the active area they show $\Delta_{\text {Min-Max }}=130 \mu \mathrm{m}$, which also fulfills the requirements for drift panels.

A reason for this deepening is the stiffback. By looking closely at figure 13 it can be seen that it goes up in the center. During the gluing process this side is turned face down and since

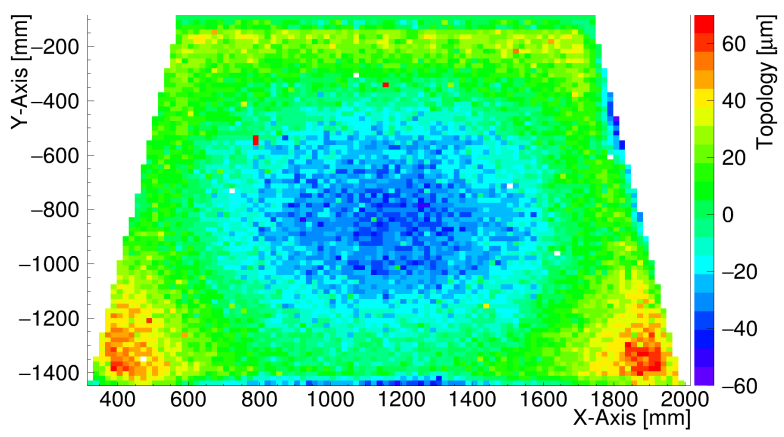

Fig. 14. Topology of the first side of a test panel. The second side faces the granite table and is sucked down with vacuum.

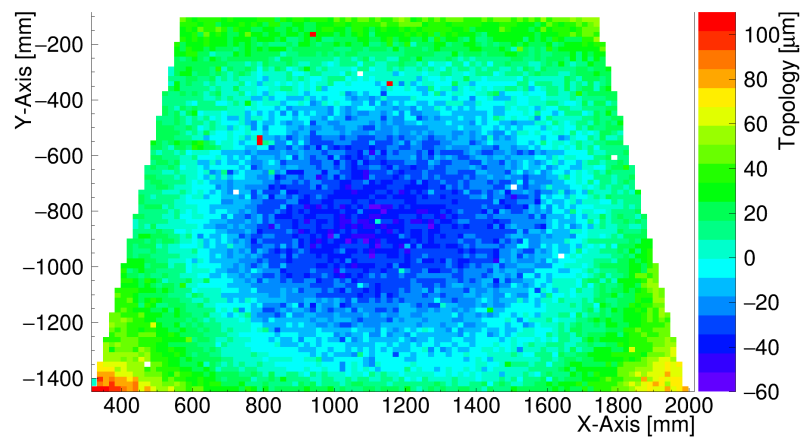

Fig. 15. Topology of the second side of a test panel. The first side faces the granite table and is sucked down with vacuum. The plot looks very similar to the one of the first side, since the deformation is small. The side facing the granite table is pressed flat with vacuum and therefore the total non planarity is transferred to the side facing the laser.

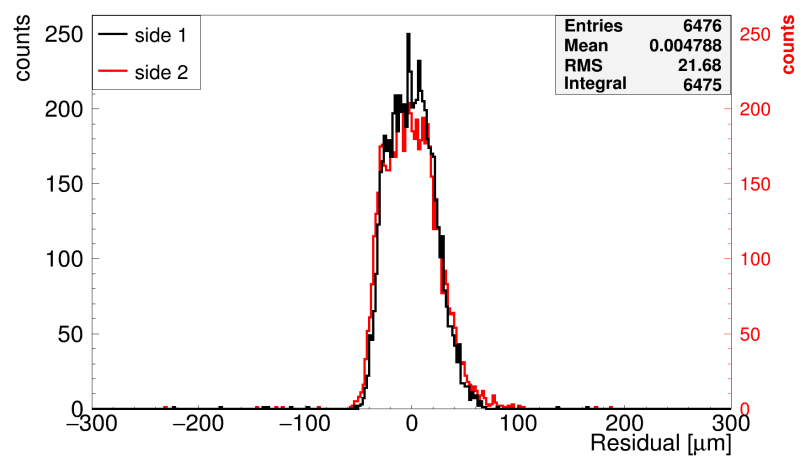

Fig. 16. Superimposed heights of both sides. The RMS is in both cases below $25 \mu \mathrm{m}$ with $\Delta_{M i n-M a x}=130 \mu \mathrm{m}$ for the active area. The results meet the design requirements of the collaboration.

the stiffback is not perfectly rigid it can bend a bit which increases the rise in the center of the stiffback. The measured panel topology is therefore the negative of the stiffback. To get rid of this issue the stiffback is pulled upwards in the center during glue curing of the second step.

\section{Interconnections}

Even if the panels come out perfectly of the gluing process, there can still be deformations during operation of the detec- 
tors due to overpressure of the operating gas mixture inside. To ensure a constant renewing of the detector gas and therefore to prevent contamination with air the detectors are operated at $2-3$ mbar overpressure which results in a blow up of the chambers. By placing screws through the quadruplet, so called interconnections, this blow up can be minimized. Figure 17 shows a simulation of the blow up of a panel with six interconnects [8]. The simulation was done using the finite element method (FEM) code ANSYS [9].

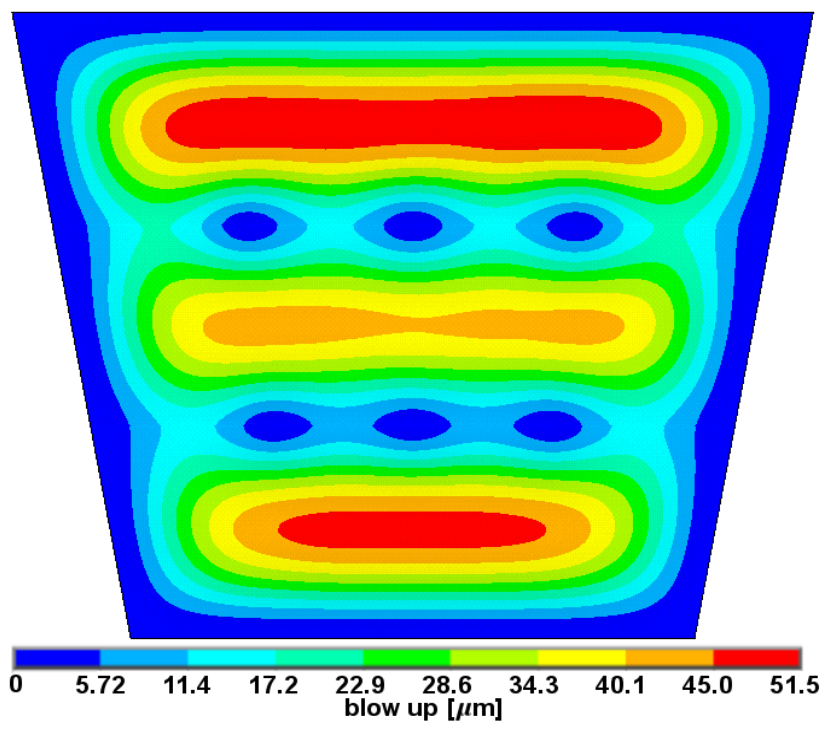

Fig. 17. Simulation of panel blow up due to 3 mbar overpressure. Six interconnections minimize the maximal deformation to $52 \mu \mathrm{m}$. The position of the interconnections will change slightly for the mass production.

A perfectly flat panel would show a deformation of $\Delta_{\text {Min-Max }}=52 \mu \mathrm{m}$ with 2 mbar overpressure.

The current design foresees also six interconnections but at slightly different positions than shown in figure 17. The reason is, that some interconnections will also be used as fixation for global optical alignment platforms, to monitor the positions of different quadruplets to each other.

\section{CONCLUSION}

We presented the construction method of large area Micromegas, which will in combination with sTGC detectors replace the current technologies in the inner endcap region of the ATLAS experiment.

The disk-like shape of the endcap is divided in eight small and eight large sectors. Each of the sectors is again divided in radial direction into two trapezoidal shaped geometries. Four detector layers are combined to form a quadruplet of Micromegas for an improved spatial resolution. A quadruplet consists of five sandwich panels with either readout structure or a copper cathode on their surfaces. The panels are built in a two step gluing procedure. Required precision is achieved using a flat granite table and a flat lightweight rigid structure, called stiffback. Since industry is not able to produce PCBs in the dimensions required for the project, the active area of one SM2 trapezoid is made up of three PCBs. A precise alignment frame takes care of positioning the boards correctly to each other to achieve the required parallelism of the readout structure.

A fast laser triangulation system is used to check the surface quality of the produced panels. The results show, that the described methods work and that the requirements can be met. The stiffback has an RMS of $14.3 \mu \mathrm{m}$, the panels have an RMS below $25 \mu \mathrm{m}$. Methods to further improve the planarity are invented but need to be tested.

Results of an ANSYS simulation show, that the blow up of a quadruplet due to 3 mbar overpressure during operation can be minimized to $\Delta_{M i n-M a x}=52 \mu \mathrm{m}$ using six screws interconnecting the quadruplet inside its active area.

\section{ACKNOWLEDGMENT}

We acknowledge the support by the DFG Excellence Cluster Universe.

\section{REFERENCES}

[1] ATLAS collaboration, The ATLAS Experiment at the CERN Large Hadron Collider JINST P08003 3 (2008)

[2] Y. Giomataris et al., MICROMEGAS: a high-granularity positionsensitive gaseous detector for high particle-flux environments Nuclear Instruments and Methods in Physics Research Section A, Volume 376 (1996): 29 - 35

[3] V. Smakhtin et al., Thin Gap Chamber upgrade for SLHC: Position resolution in a test beam Nuclear Instruments and Methods in Physics Research Section A, Volume 598 (2009): 196 - 200

[4] ATLAS Collaboration, Performance of the ATLAS Trigger System in 2010 The European Physical Journal C, Volume 72:1849 (2012)

[5] ATLAS Collaboration, ATLAS New Small Wheel Technical Design Report ATLAS - TDR-20-2013 (2013) Available: https://cds.cern.ch/record/1552862/files/ATLAS-TDR-020.pdf

[6] T. Alexopoulos et al., A spark-resistant bulk-micromegas chamber for high-rate applications Nuclear Instruments and Methods in Physics Research Section A, Volume 640 (2011): 110 - 118

[7] MICRO-EPSILON, OPTO NCDT $2300 \quad B L$ Datasheet http://www.micro-epsilon.de/download/products/_laser-sensor/ dax--optoNCDT-2300BL--en.html

[8] Elias Pree, Construction of Large Area Micromegas Detectors LMU Munich, Master Thesis, 2014

[9] ANSYS, Inc., ANSYS Mechanical APDL Element Reference http://148.204.81.206/Ansys/150/ANSYS\%20Mechanical\%20APDL\% 20Element\%20Reference.pdf 